Microwave and

Optical Technology Letters

\title{
Multilayer bandpass filter-polarizer with stopband extension far beyond twofold center frequency
}

\begin{tabular}{|r|l|}
\hline Journal: & Microwave and Optical Technology Letters \\
\hline Manuscript ID & Draft \\
\hline Wiley - Manuscript type: & Research Article \\
\hline Date Submitted by the Author: & n/a \\
\hline Complete List of Authors: & $\begin{array}{l}\text { Belyaev, Boris; Kirensky Institute of Physics, ; Reshetnev Siberian State } \\
\text { Aerospace University, } \\
\text { Tyurnev, Vladimir; Kirensky Institute of Physics, }\end{array}$ \\
\hline Keywords: & Filter, polarizer, frequency selective surface, grating, multilayer design \\
\hline \multicolumn{2}{|c}{} \\
\hline
\end{tabular}

SCHOLARONE $^{\text {IM }}$

Manuscripts 


\title{
Multilayer bandpass filter-polarizer with stopband extension far beyond twofold center frequency
}

\author{
B. A. Belyaev ${ }^{1,2,3}$ | \\ V. V. Tyurnev ${ }^{1,2}$ \\ ${ }^{1}$ Kirensky Institute of Physics, Federal Research Center KSC SB RAS, Krasnoyarsk 660036, Russia \\ ${ }^{2}$ Siberian Federal University, Krasnoyarsk 660041, Russia \\ ${ }^{3}$ Siberian State Aerospace University, Krasnoyarsk 660014, Russia \\ Correspondence \\ V. V. Tyurnev, Kirensky Institute of Physics, Federal Research Center KSC SB RAS, Krasnoyarsk \\ 660036, Russia. \\ Email: tyurnev@iph.krasn.ru
}

\begin{abstract}
We propose a multilayer bandpass filter in which every resonator consists of two identical dielectric layers separated by a grating of parallel strip conductors. The resonators themselves are separated from each other and from the ambient space by gratings of strip conductors with an axis orthogonal to the axis of the gratings inside the resonators. The proposed filter construction acts at the same time as a polarizer. The device frequency response computed with use of the provided simple formulas is in a good qualitative agreement with numerical electromagnetic analysis of its 3D model.
\end{abstract}

\section{KEYWORDS}

Filter, polarizer, frequency selective surface, grating, multilayer design

\section{1 | INTRODUCTION}

Recently, a novel multilayer bandpass filter with greatly extended lower and upper stopbands was proposed [1]. It is composed of resonant dielectric layers, which are separated from each other and from the ambient space by nonresonant gratings of the parallel strip conductors. The gratings act as mirrors with the required reflectivity, and their axes are parallel to each other. Every dielectric layer has a nearly equidistant spectrum of the resonant frequencies at which the optical thickness is approximately multiple to a half wavelength. Therefore, the upper stopband of the filter is limited from above at a frequency of about $2 f_{0}$, where $f_{0}$ is the center frequency of the passband. However, the structure [1] ceases to act as a filter and becomes almost transparent within a wide frequency range including the stopbands if the electric field is orthogonal to the strip conductors. The formulas for synthesizing this filter were derived in [2].

In this letter, we propose a considerably improved construction of the multilayer bandpass filter with nonresonant gratings of strip conductors. The stopband in this filter may extend far beyond the limit of $2 f_{0}$ that being in the cited filter [1]. Moreover, this device acts as a polarizer, which almost fully reflects the waves when the electric field is orthogonal to the strip conductors in the grating on the filter input. We provide formulas for computing the frequency response of the filter structure. In addition, we compare the frequency responses of the filter described in [1] and the proposed filter for two orthogonal polarizations 
of the incident wave. Besides, we compare the frequency responses obtained by the formulas with results of the numerical electromagnetic simulation of the device 3D model in CST Microwave Studio package.

\section{2 | DEVICE STRUCTURE}

Figure 1 shows the proposed filter in which every resonator consists of two identical dielectric layers separated by a grating of strip conductors. The resonators themselves are separated from each other and from the ambient space also by gratings of strip conductors but with the axis orthogonal to the axis of the gratings inside the resonators. Therefore, there are two types of the alternating gratings: inter-resonator gratings (1) and intra-resonator grating (2).

In the proposed construction, nearly full reflection of the incident wave is observed only in the case when the electric field vector is parallel to the strip conductors of the intra-resonator gratings (2). In other cases, every pair of the neighboring dielectric layers separated by the intra-resonator grating (2) acts as a resonator, and every inter-resonator grating (1) acts as a coupling structure of the resonator. The function of the latter is to ensure the required coupling degree of the double-layer dielectric resonator with its neighbor or with the ambient space. The coupling degree is controlled by the reflectivity of the grating, which depends on the construction parameters [3].

At a frequency $f_{0}$, the resonators have minima of the electric field on their edges, i.e., on the interresonator gratings, and have maxima on the intra-resonator gratings. Therefore, the intra-resonator gratings act as quasi-lumped capacities $C_{1}, C_{2}$, and $C_{3}$, as depicted in the equivalent circuit in Figure 2 . As a result, they considerably lower the first resonant frequency relative to the second resonant frequency. Note that the inter-resonator gratings, acting as quasi-lumped inductances $L_{1}, L_{2}$, and $L_{3}$ (Figure 2), facilitate additional lowering of the first resonant frequency [1]. However, this additional frequency lowering is reduced as the fractional passband width increases [1]. The use of quasi-lumped capacities in order to lower the first resonant frequency of resonators is widely applicable in microwave techniques, for example, in suspended dual-mode stripline resonators [4].

We shall characterize the $k$ th inter-resonator grating with a period $T_{L k}$ and spacing between the strip conductors $s_{L k}$. It is important that the inequality $T_{L k}<0.4 \lambda$ [5] must be held, where $\lambda$ is the wavelength in the dielectric layer at $f_{0}$. This constraint suppresses the propagation in the dielectric layers of higher modes generated by the gratings and shifts the resonances of the gratings to the higher frequency range. These conditions prevent the formation of extra spurious passbands within the stopband in the frequency response of the filter [1]. In addition, this inequality is the usability condition of the quasi-static approximation for computing the near-region field of the grating [5].

Let the dielectric layers in the filter have thicknesses $h_{k}<\lambda / 4$ and all the intra-resonator gratings in the filter may have their own parameters $s_{C k}$ and $T_{C k}$. The smaller the ratio $s_{C k} / T_{C k}$ we set the greater decrease of the first resonant frequency relative to the second resonant frequency we get. The greater the ratio $s_{L k} / T_{L k}$ in the $k$ th inter-resonator grating we set the greater the coupling between the resonators and the wider filter passband we get [3].

There are optimal spacings $s_{L k}$ in the inter-resonator gratings with the given periods $T_{L k}$ when the filter fractional bandwidth $\Delta f / f_{0}$ and parameters of the intra-resonator gratings $s_{C k}$ and $T_{C k}$ are specified. The spacings $s_{L k}$ grow when $\Delta f / f_{0}$ or $T_{L k}$ increase. In all cases, the external gratings of the structure have the widest spacing. The thickness $h_{k}$ of the dielectric layer depends on $\lambda$ and spacings $s_{L k}$ and $s_{C k}$. It becomes thinner when $s_{L k}$ or $s_{C k}$ becomes narrower.

In the equivalent circuit of the multilayer filter with the crossed gratings of strip conductors in Figure 2, the dielectric layers are represented as cascaded transmission-line sections with electrical lengths $\theta_{k}$. This circuit describes all the main selective properties of the filter. 


\section{3 | ANALYSIS FORMULAS}

The frequency dependences of the transmission and reflection coefficients (i.e. frequency response) of a filter are usually characterized by the scattering matrix $\mathbf{S}(f)$, which relates the normalized electric strength amplitudes of outgoing waves $\left(b_{k}\right)$ with the analogous amplitudes of incoming waves $\left(a_{k}\right)$ on both sides (port 1 and port 2) by the equation [6]

$$
\left(\begin{array}{l}
b_{1} \\
b_{2}
\end{array}\right)=\mathbf{S}\left(\begin{array}{l}
a_{1} \\
a_{2}
\end{array}\right) .
$$

Here, amplitudes $a_{k}$ and $b_{k}$ are normalized such that the total power $P_{k}$ coming into port $k$ per unit area is expressed by the equation

$$
P_{k}=\frac{1}{2}\left|a_{k}\right|^{2}-\frac{1}{2}\left|b_{k}\right|^{2}
$$

The scattering matrix $\mathbf{S}$ of any multilayer structure may be easily computed if the transfer matrices $\mathbf{M}$ for every component of the structure are known. Matrix $\mathbf{M}$ is also called the ABCD matrix or characteristic matrix $[6,7]$. It relates the tangential components of the electric and magnetic field strengths on the first and second surfaces of a planar structure by the following equation:

$$
\left(\begin{array}{c}
E_{1} \\
Z_{0} H_{1}
\end{array}\right)=\mathbf{M}\left(\begin{array}{c}
E_{2} \\
Z_{0} H_{2}
\end{array}\right)
$$

where the free space characteristic impedance is $Z_{0}=\left(\mu_{0} / \varepsilon_{0}\right)^{1 / 2}$.

Matrices $\mathbf{M}$ and $\mathbf{S}$ are related by the formulas [6]

$$
\begin{gathered}
\mathbf{M}=\left(\begin{array}{cc}
\frac{1+S_{11}-S_{22}-\operatorname{det}\left[S_{i k}\right]}{2 S_{21}} \sqrt{\frac{n_{2}}{n_{1}}} & \frac{1+S_{11}+S_{22}+\operatorname{det}\left[S_{i k}\right]}{2 S_{21} \sqrt{n_{1} n_{2}}} \\
\frac{1-S_{11}-S_{22}+\operatorname{det}\left[S_{i k}\right]}{2 S_{21}} \sqrt{n_{1} n_{2}} & \frac{1-S_{11}+S_{22}-\operatorname{det}\left[S_{i k}\right]}{2 S_{21}} \sqrt{\frac{n_{1}}{n_{2}}}
\end{array}\right), \\
\mathbf{S}=\left(\begin{array}{cc}
1-2 \frac{M_{21}+M_{22} n_{2}}{d} & 2 \frac{\sqrt{n_{1} n_{2}}}{d} \operatorname{det}\left[M_{i k}\right] \\
2 \frac{\sqrt{n_{1} n_{2}}}{d} & 1-2 \frac{M_{11} n_{1}+M_{21}}{d}
\end{array}\right), d \equiv M_{11} n_{1}+M_{12} n_{1} n_{2}+M_{21}+M_{22} n_{2}
\end{gathered}
$$

where $n_{1}$ and $n_{2}$ are the refractive indices of the media that neighbor the planar structure.

The transfer matrix of the $k$ th dielectric layer has the form [7]

$$
\mathbf{M}=\left(\begin{array}{cc}
\cos \theta_{k} & \frac{-i}{n_{k}} \sin \theta_{k} \\
-i n_{k} \sin \theta_{k} & \cos \theta_{k}
\end{array}\right), \theta_{k} \equiv \frac{2 \pi f}{c} n_{k} h_{k}
$$

where $c$ is the velocity of light, $n_{k}$ is the refractive index of the dielectric layers, $h_{k}$ is the thickness of the $k$ th layer, and $i$ is the imaginary unit, as found in the time-dependent factor $\exp (-i 2 \pi f t)$.

Using the formulas for the reflection and transmission coefficients that were derived in [3] with the use of the quasi-static approximation, we write the scattering matrix of the inter-resonator grating as 


$$
\mathbf{S}^{L}=\left(\begin{array}{cc}
\frac{n_{1}-n_{2}-i \Phi}{n_{1}+n_{2}+i \Phi} & \frac{2 \sqrt{n_{1} n_{2}}}{n_{1}+n_{2}+i \Phi} \\
\frac{2 \sqrt{n_{1} n_{2}}}{n_{1}+n_{2}+i \Phi} & \frac{-n_{1}+n_{2}-i \Phi}{n_{1}+n_{2}+i \Phi}
\end{array}\right), \Phi \equiv c /\left[f T \ln \sec \left(\frac{\pi s_{L}}{2 T}\right)\right]
$$

and the scattering matrix of the intra-resonator grating as

$$
\mathbf{S}^{C}=\left(\begin{array}{cc}
\frac{n_{1}-n_{2}+i \Psi}{n_{1}+n_{2}-i \Psi} & \frac{2 \sqrt{n_{1} n_{2}}}{n_{1}+n_{2}-i \Psi} \\
\frac{2 \sqrt{n_{1} n_{2}}}{n_{1}+n_{2}-i \Psi} & \frac{-n_{1}+n_{2}+i \Psi}{n_{1}+n_{2}-i \Psi}
\end{array}\right), \Psi \equiv 2 f T \frac{n_{1}^{2}+n_{2}^{2}}{c} \ln \csc \left(\frac{\pi s_{C}}{2 T}\right) .
$$

It should be borne in mind that formulas (7) and (8) were derived for the case of the gratings with infinitely thin ideal strip conductors.

The transfer matrix of an entire multilayer structure is equal to the product of the transfer matrices for each component of the structure [6,7]. Using formulas (4) through (7), we may compute the frequency response of the proposed filter and therefore we may adjust the filter to get the required frequency response.

\section{4 | FREQUENCY RESPONSE}

As an example we designed the fifth order filter with the center frequency $f_{0}=1 \mathrm{THz}$, the fractional bandwidth $\Delta f / f_{0}=10 \%$, and the reflection maxima in the passband of $-14 \mathrm{~dB}$. As a material of the dielectric layers we used polymethylpentene with the refractive index $n=1.46$. We supposed that all the dielectric layers have the same thickness $h_{k}=24 \mu \mathrm{m}$ and all the gratings have the same period $T_{L k}=T_{C k}=40$ $\mu \mathrm{m}$. At that rate, it was necessary to fit only the spacings in the gratings using the universal rules of a filter optimization $[8,9]$ in order to get the optimal couplings and the tuned resonant frequencies of the resonators. The optimal spacing values in micrometers are presented in Table 1 in the line "formulas".

In Figure 3, the solid line (a) shows the frequency dependence of the transmission coefficient $\left|S_{21}\right|^{2}$ computed by the presented formulas for the multilayered filter with the crossed gratings of the strip conductors when the electric field of the incident wave is parallel to the strip conductors in the external gratings. The upper stopband of the filter extends up to $3.6 f_{0}$ at a transmission level of $-30 \mathrm{~dB}$, while the lower stopband extends down to DC.

For comparison, the dash line $(b)$ in Figure 3 shows the corresponding frequency dependence for the similar filter without the intra-resonator gratings that is mentioned above [1]. In that filter, the upper stopband extends only up to frequency of $1.9 f_{0}$ at a transmission level of $-30 \mathrm{~dB}$.

In order to estimate the accuracy of the presented formulas, we also synthesized the same filter with the same passband parameters. Instead of the formulas, we used the numerical electromagnetic simulation of the 3D model in CST Microwave Studio package. We assumed that the conductors in all the gratings are made of cooper with the conductivity of $5.96 \times 10^{7} \mathrm{~S} / \mathrm{m}$ and have the thickness of $0.1 \mu \mathrm{m}$. A dashdot line $(c)$ in Fig. 3 depicts the simulated frequency dependence of the transmission coefficient for this case while the obtained structure parameters are presented in Table 1 in line "CST MS". One can see good agreement between the curves $(a)$ and $(c)$ in the wide frequency range. However, substitution of the ideal conductors for the real conductors results in ohmic loss in the filter passband about $1.5 \mathrm{~dB}$.

In Figure 4, the frequency dependences of the transmission coefficient $\left|S_{21}\right|^{2}$ and the reflection coefficient $\left|S_{11}\right|^{2}$ for the same filter but in a narrow frequency range near the passband are presented. The dot 
line shows the transmission coefficient computed by the formulas for the gratings with infinitely thin ideal strip conductors and the spacing values presented in Table in line "formulas". The solid lines show the transmission and reflection coefficients simulated in CST Microwave Studio package for the gratings with the copper strip conductors of $0.1 \mu \mathrm{m}$ thickness and the spacing values in Table in line "CST MS". The dash line show the transmission coefficient simulated in CST Microwave Studio package for the gratings with the copper strip conductors of $0.1 \mu \mathrm{m}$ thicknesses but with the spacing values presented in Table 1 in line "formulas". One can see a qualitative agreement between the quasi-static computation by the proposed formulas and the numerical electromagnetic simulation of the 3D filter model. That proves the validity of the proposed formulas. A small lowering of the center frequency of the filter passband is due to the simplified grating model with the infinitely thin ideal strip conductors that underlies the formulas (7) and (8).

\section{5 | CONCLUSION}

Thus, the proposed novel construction of the multilayered bandpass filter is at the same time a polarizer. In this construction, all the resonators are separated from each other and from the ambient space by the gratings of the parallel strip conductors while each of the resonators consists of two equal dielectric layers that are also separated by a grating of strip conductors but with the axis orthogonal to the axis of the inter-resonator gratings. It was shown a qualitative agreement between the formulaic quasi-static computation for the simplified model of the filter-polarizer and the numerical electromagnetic simulation of the device 3D model. This fact greatly simplifies the process of the parametric synthesis of the filter-polarizer with the specified frequency response because the initial structure parameters may be easy obtained by the presented formulas.

\section{ACKNOWLEDGMENT}

This work was supported by Ministry of Education and Science of the Russian Federation while realizing a complex project to create high-tech production, contract No 03.G25.31.0279. 


\section{REFERENCES}

1. B. A. Belyaev and V. V. Tyurnev, Multilayer bandpass filter with extended lower and upper stop bands, Optics Letters 40 (2015), 4333-4345.

2. B. A. Belyaev and V. V. Tyurnev, Design of bandpass filters composed of dielectric layers separated by gratings of strip conductors, Optics Letters, 41 (2016) 536-539.

3. B. A. Belyaev and V. V. Tyurnev, Diffraction of electromagnetic waves on a one-dimensional strip conductor grating located at the interface between dielectric media, Russian Physics J., 58 (2015), 646657.

4. B. A. Belyaev, A. A. Leksikov, A. M. Serzhantov, and V. V. Tyurnev, Highly selective suspended stripline dual-mode filter, Prog Electromagn Res Lett., 25 (2011), 57-66.

5. L. A. Vainshtein, Diffraction of electromagnetic waves by the gratings of parallel conducting strips, Zhurnal Tekhnicheskoy Fiziki, 25 (1955), 847-852 (In Russian).

6. K. C. Gupta, R. Garg, and R. Chadha, Computer-aided design of microwave circuits, Artech House, 1981.

7. H. A. Macleod, Thin-film optical filters, CRC Press, 2010.

8. B. A. Belyaev, V. V. Tyurnev, and V. F. Shabanov, One-dimensional photonic crystal bandpass filters, Doklady Physics, 59 (2014), 73-78.

9. B. A. Belyaev, V. V. Tyurnev, The method for microstrip filters parametric synthesis, Int. Crimean Conf. "Microwave and Telecommunication Technology", CriMiCo'2006, Sevastopol, p. 517-519 (2006). 
1

2

3

4

5

6

7

8

9

10

11

12

13

14

15

16

17

18

19

20

21

22

23

24

25

26

27

28

29

30

31

32

33

34

35

36

37

38

39

40

41

42

43

44

45

46

47

48

49

50

51

52

53

54

55

56

57

58

59

60

TABLE 1. Optimal spacing values

\begin{tabular}{|l|c|c|c|c|c|c|}
\hline & $s_{L 1}$ & $s_{L 2}$ & $s_{L 3}$ & $s_{C 1}$ & $s_{C 2}$ & $s_{C 3}$ \\
\hline formulas & 37.94 & 21.96 & 20.32 & 4.332 & 2.164 & 2.096 \\
\hline CST MS & 38.50 & 23.10 & 21.69 & 5.180 & 2.94 & 2.875 \\
\hline
\end{tabular}




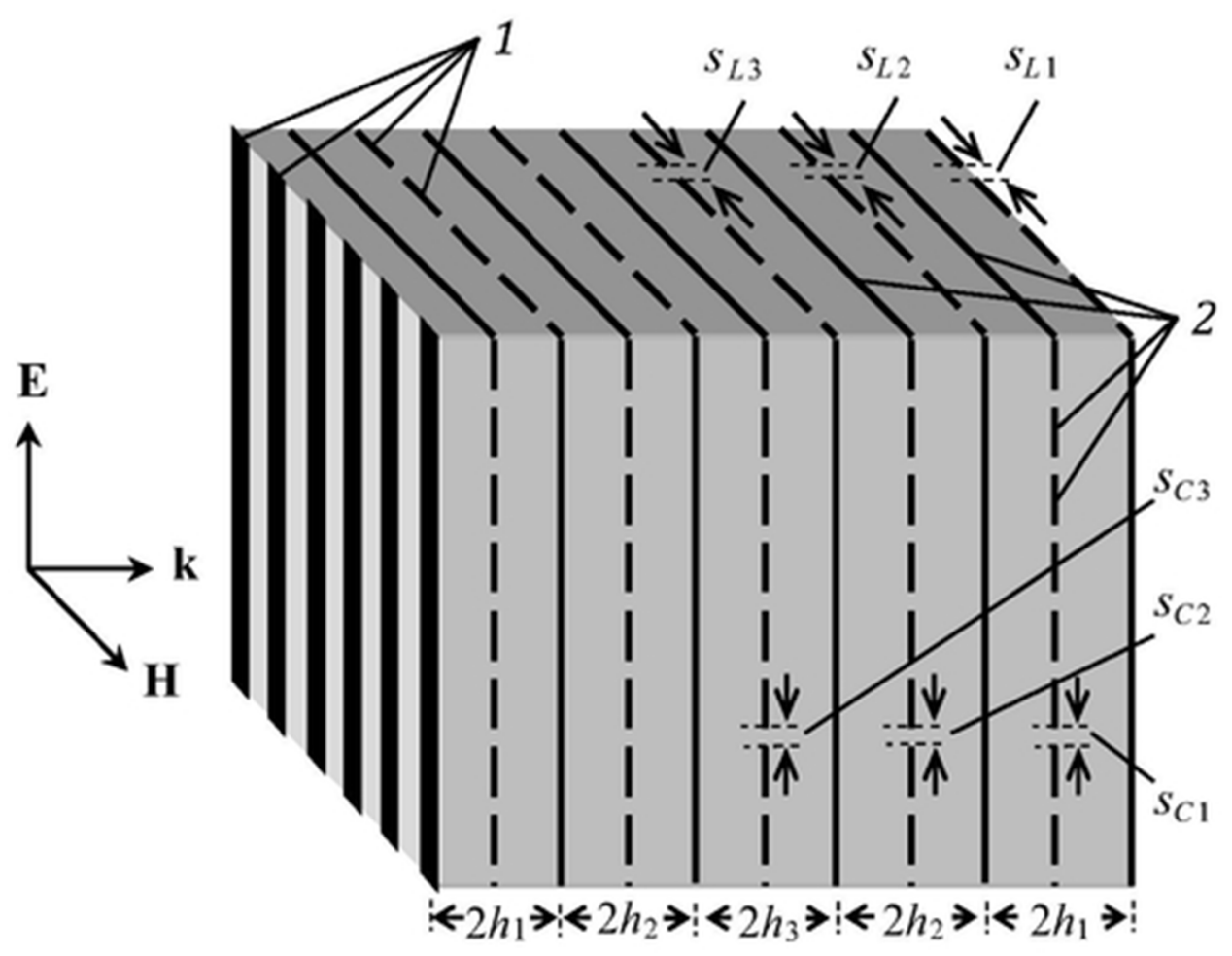

Figure 1. Multilayer filter with crossed gratings of strip conductors. (Dashes show the cross sections of the strip conductors and continuous lines show the longitudinal sections of the strip conductors).

$40 \times 31 \mathrm{~mm}(300 \times 300 \mathrm{DPI})$ 


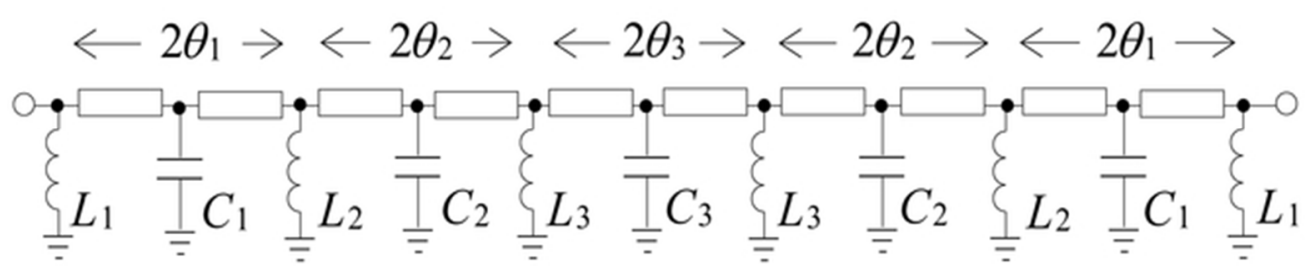

Figure 2. Equivalent circuit of multilayer filter with crossed gratings of strip conductors.

$56 \times 11 \mathrm{~mm}(300 \times 300$ DPI $)$ 


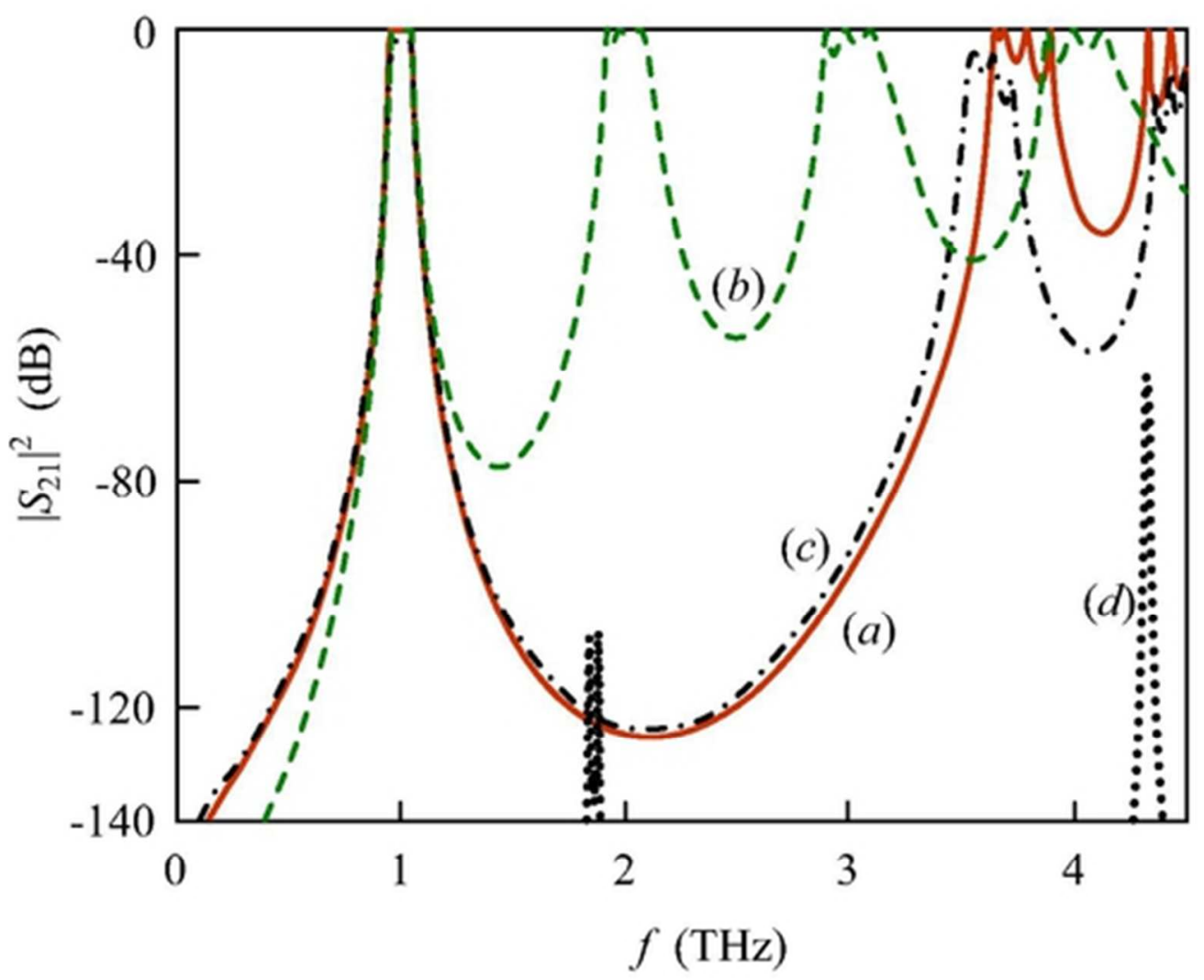

Figure 3. Frequency dependences of transmission coefficient in wide frequency range computed by formulas (a), (b) and simulated in CST Microwave Studio (c), (d) for filter with intra-resonator gratings (a), (c), and (d) and for filter without the latters (b).

$42 \times 34 \mathrm{~mm}(300 \times 300 \mathrm{DPI})$ 
39

40

41

42

43

44

45

46

47

48

49

50

51

52

53

54

55

56

57

58

59

60

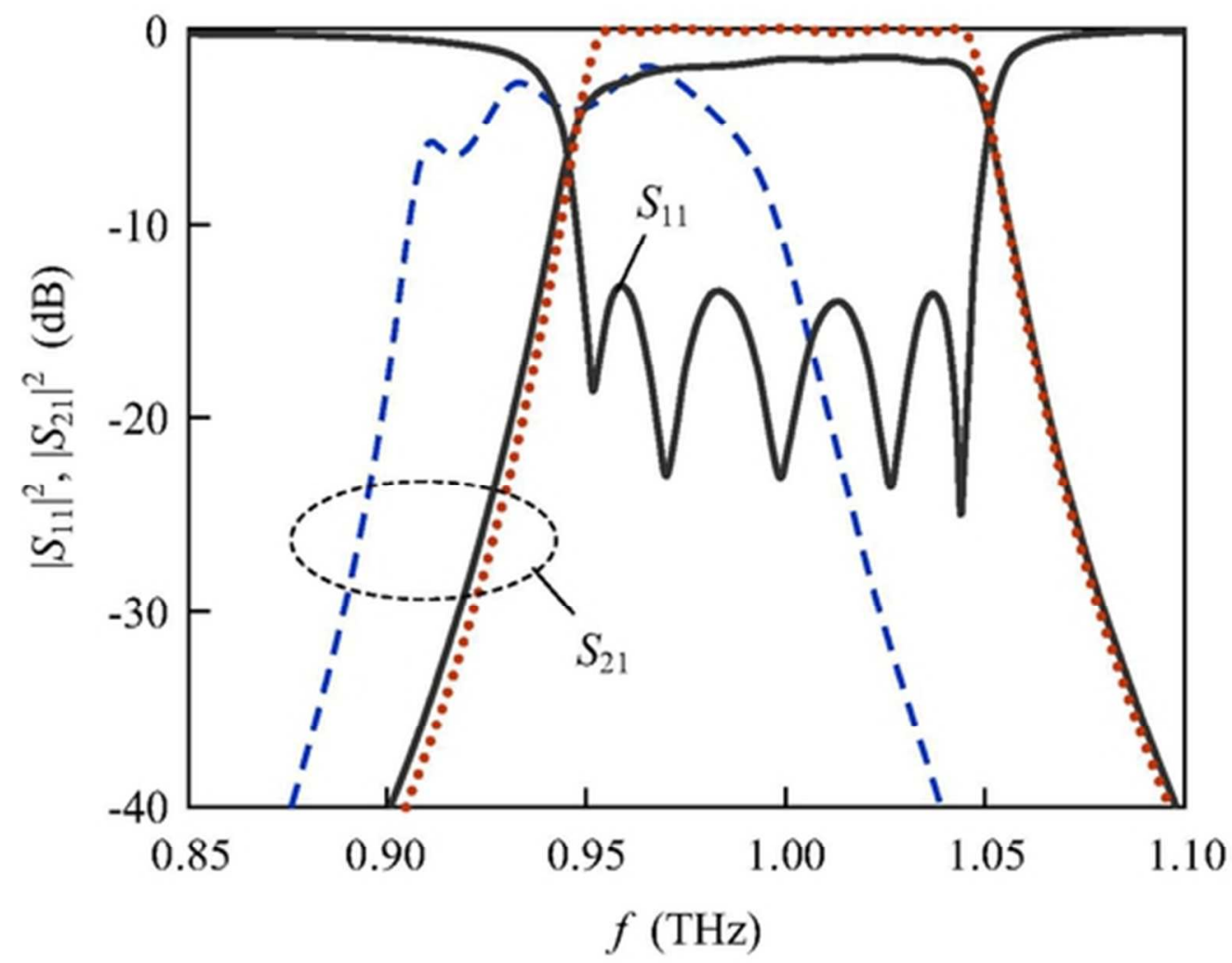

Figure 4. Frequency response of multilayer filter with crossed gratings of strip conductors in narrow frequency range (Solid and dash lines were simulated in CST Microwave Studio package for the values in Table 1 in lines "CST MW" and "formulas", respectively, while dot line was computed by the formulas)

$42 \times 33 \mathrm{~mm}(300 \times 300 \mathrm{DPI})$ 